\title{
Fault Tolerant Controller Design for a Faulty UAV Using Fuzzy Modeling Approach
}

\author{
Moshu Qian, ${ }^{1,2}$ Ke Xiong, ${ }^{2}$ Lili Wang, ${ }^{3}$ and Zhongmin Qian ${ }^{4}$ \\ ${ }^{1}$ Key Laboratory of UAV Technology (Nanjing University of Aeronautics and Astronautics), Ministry of Industry \\ and Information Technology, Nanjing 210016, China \\ ${ }^{2}$ State Key Laboratory of Mechanics and Control of Mechanical Structures, Nanjing University of Aeronautics \\ and Astronautics, Nanjing 210016, China \\ ${ }^{3}$ Material and Technical Process Assurance Center, Beijing Spacecrafts, Beijing 100190, China \\ ${ }^{4}$ College of Computer Science and Technology, Nanjing University of Aeronautics and Astronautics, Nanjing 210016, China \\ Correspondence should be addressed to Moshu Qian; qianmoshu@nuaa.edu.cn
}

Received 16 April 2016; Revised 14 August 2016; Accepted 24 August 2016

Academic Editor: Asier Ibeas

Copyright (C) 2016 Moshu Qian et al. This is an open access article distributed under the Creative Commons Attribution License, which permits unrestricted use, distribution, and reproduction in any medium, provided the original work is properly cited.

\begin{abstract}
We address a fault tolerant control (FTC) issue about an unmanned aerial vehicle (UAV) under possible simultaneous actuator saturation and faults occurrence. Firstly, the Takagi-Sugeno fuzzy models representing nonlinear flight control systems (FCS) for an UAV with unknown disturbances and actuator saturation are established. Then, a normal $H$-infinity tracking controller is presented using an online estimator, which is introduced to weaken the saturation effect. Based on the normal tracking controller, we propose an adaptive fault tolerant tracking controller (FTTC) to solve actuator loss of effectiveness (LOE) fault problem. Compared with previous work, this approach developed in our research need not rely on any fault diagnosis unit and is easily applied in engineering. Finally, these results in simulation indicate the efficiency of our presented FTC scheme.
\end{abstract}

\section{Introduction}

UAV is a class of aircraft without a human pilot onboard. The flight of an UAV, depending on flight control systems that include actuator, sensor, and so on, is controlled autonomously either by onboard computers or by the remote control of a pilot [1]. It is a quickly time-varying and strong coupling characteristic of complex nonlinear system [2]. As a class of aerial vehicle without human, a huge number of system components including actuators, sensors, or others inevitably cause various faults of flight control systems in an UAV. Moreover, different from manned aerospace vehicles, an unmanned aerial vehicle allows for a wider range of flight operating points that may lead to more vulnerability of failures [3]. To enhance the safety and reliability, a fault accommodation method for UAV is taken into account when one designs the flight control systems [4]. Because the flight control systems of UAV are nonlinear systems, the traditional control method is not appropriate. However,
T-S fuzzy modeling is an effective tool which connects linear control system with nonlinear one [5], which has been an active research topic. It can utilize a series of local linearized models to realize the global approximation of an arbitrary nonlinear smooth system function; then the complex analysis and application of nonlinear control system are greatly simplified, which is also its main merit. For the above reason, a T-S fuzzy system describing nonlinear FCS of UAV is obtained in this paper. On the other hand, although some results about fault accommodation for fuzzy control system have been achieved in recent years, the FTC problem in an actual control system still exists and needs solving; for example, the FTTC design of a Takagi-Sugeno fuzzy system under disturbance, actuator loss of effectiveness (LOE) faults, and saturation simultaneously is a challenging public problem now. In [6] the problem of output tracking for nonlinear studied object under actuator faults is investigated; then the existence condition of interval fuzzy $\mathrm{H}$-infinity tracking control is obtained. A FTC approach based on 
an observer is proposed for a T-S fuzzy model with actuator loss of effectiveness faults in [7]; it guarantees the stability of a controlled object in actuator faulty case. In [8], the problem of FTTC is discussed for a class of fuzzy system with premise unmeasurable factors; the fuzzy descriptor observer is addressed for estimating the sensor faults. In [9], an active FTTC approach is researched for the nonlinear system with sensor faults via T-S multiple models. Reference [10] studies a FTC scheme of a fuzzy control system under actuator LOE faults using delta operator approach, which compensates for the function of actuator faults and makes T-S fuzzy system stable. In [11], the sufficient condition for a fuzzy control system under actuator LOE faults is derived from the scaled gain theory, which achieves its stochastic stability and satisfactory $H_{\infty}$ performance. However, the actuator saturation is not discussed in [6-26], which certainly exists and needs solving in actual FCS, so the fault accommodation results obtained above have some limitations in dealing with two kinds of actual actuator faults simultaneously. In [27], the authors address the problem of actuator saturation and loss of effectiveness faults for singular T-S fuzzy control systems; a passive regulator design is solved by the linear matrices inequalities and the ultimate stability is proved. It is worth noting that the unknown disturbance is not studied in [27], which is inevitable in the actual FCS of an unmanned aerial vehicle. So fault tolerant tracking controller design problems of nonlinear system expressed by fuzzy models have not been thoroughly solved, which is still a great challenge.

As previously discussed, we focus on the FTTC design for nonlinear flight control systems of UAV under unknown disturbances, actuator saturation constraints, and actuator LOE faults. The novelty of our research compared with existing work includes the following key-points:

(1) Actuator saturation constraints and unknown disturbances represented by T-S fuzzy model are considered; the adaptive approach can guarantee asymptotic tracking control performance of the nonlinear closeloop flight control systems.

(2) Adding actuator faults to above T-S fuzzy model, a novel fault tolerant control design including an adaptive fault estimator is given to accommodate the loss of effectiveness faults.

Therefore, our results obtained in this paper can be regarded as the complements of previous research. When actuator faults occur in an actual UAV, the FTC scheme of this paper guarantees the asymptotical tracking of FCS. Finally, simulation results show that our design approach has the favorable fault tolerant capability and robust ability.

\section{Problem Formation}

2.1. The Nonlinear Dynamics for $U A V$. The flight control systems of UAV adapted in this study involve the aerodynamic performance brought from control surfaces. All control inputs are fully independent and shown in Figure 1. $\delta_{\text {th }}$ is the throttle; $\delta_{\mathrm{ar}}, \delta_{\mathrm{al}}, \delta_{\mathrm{fr}}, \delta_{\mathrm{fl}}, \delta_{\mathrm{er}}, \delta_{\mathrm{el}}$ are the right aileron and left one, similarly, followed by flap, the right ruddervator

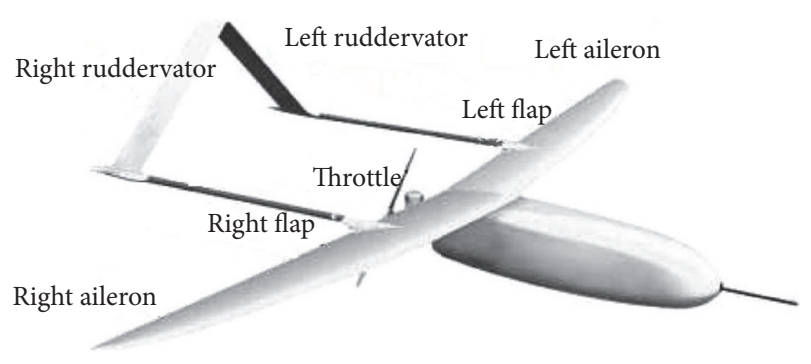

FIgUre 1: The UAV Aerosonde.

(V inverted tail). As flight control systems are open-loop unstable and few redundancies can be offered by the other controls, control surface faults addressed above would be very serious [4].

The following dynamics of the UAV is proposed for a rigid-body UAV with a fixed centre of gravity (c.g.).

$$
\begin{aligned}
& \dot{u}=-v r-w q+\frac{F_{x}}{M} \\
& \dot{v}=-u r+w p+\frac{F_{y}}{M} \\
& \dot{w}=-v p+u q+\frac{F_{z}}{M},
\end{aligned}
$$

where the weight $M$ is a constant, forces $F_{x}, F_{y}, F_{z}$ are expressed in the body frame where the linear velocity is $\mu=$ $(u, v, w)$, and $\Omega=(p, q, r)$ denote the angular velocities of roll, pitch, and yaw, respectively. Forces $F_{x}, F_{y}, F_{z}$ are due to gravity $F_{g}$, propulsion $F_{p}$, and aerodynamic effects $F_{a}$. To be more clear, in reference frame the forces are redefined as follows:

$$
\begin{aligned}
& F_{g}=\left(\begin{array}{lll}
0 & 0 & g
\end{array}\right)^{T} \\
& F_{p}=\left(\begin{array}{lll}
\frac{\bar{k} \bar{\rho}}{V} \delta_{x} & 0 & 0
\end{array}\right)^{T} \\
& F_{a}=-\left(\begin{array}{lll}
C_{D} & -C_{y} & C_{L}
\end{array}\right)^{T} S \bar{q} .
\end{aligned}
$$

Reference [4] gives the model about the propeller engine. $g$ is the acceleration of gravity, air density is represented by $\bar{\rho}, \bar{q}=1 / 2 \bar{\rho} V^{2}$ denotes aerodynamic pressure, $S$ denotes a reference surface, and $\bar{k}$ is a constant characteristic of the propeller engine. The coefficients of force are denoted by linear combination of control input and state. The relevant coefficients are

$$
\begin{aligned}
C_{D}= & C_{D 0}+\frac{S}{\pi \bar{b}^{2}} C_{L}^{2}+C_{D \delta_{\mathrm{ar}}} \delta_{\mathrm{ar}}+C_{D \delta_{\mathrm{al}}} \delta_{\mathrm{al}}+C_{D \delta_{\mathrm{fr}}} \delta_{\mathrm{fr}} \\
& +C_{D \delta_{\mathrm{fl}}} \delta_{\mathrm{fl}}+C_{D \delta_{\mathrm{er}}} \delta_{\mathrm{er}}+C_{D \delta_{\mathrm{el}}} \delta_{\mathrm{el}}
\end{aligned}
$$




$$
\begin{aligned}
C_{y}= & C_{y \beta} \beta+C_{y \delta_{\mathrm{ar}}} \delta_{\mathrm{ar}}+C_{y \delta_{\mathrm{al}}} \delta_{\mathrm{al}}+C_{y \delta_{\mathrm{fr}}} \delta_{\mathrm{fr}}+C_{y \delta_{\mathrm{fl}}} \delta_{\mathrm{fl}} \\
& +C_{y \delta_{\mathrm{er}}} \delta_{\mathrm{er}}+C_{y \delta_{\mathrm{el}}} \delta_{\mathrm{el}} \\
C_{L}= & C_{L \delta_{\mathrm{al}}} \delta_{\mathrm{al}}+C_{L \delta_{\mathrm{fl}}} \delta_{\mathrm{fl}}+C_{L \delta_{\mathrm{el}}} \delta_{\mathrm{el}}+C_{L \alpha} \alpha+C_{L 0} \\
& +C_{L \delta_{\mathrm{fr}}} \delta_{\mathrm{fr}}+C_{L \delta_{\mathrm{er}}} \delta_{\mathrm{er}}+C_{L \delta_{\mathrm{ar}}} \delta_{\mathrm{ar}}
\end{aligned}
$$

where $\bar{c}, V, \bar{b}$, and $\beta$ are the mean aerodynamic chord, airspeed, wing span, and sideslip angle, respectively. $\alpha$ denotes the angle of attack.

The relationships between the angular velocities, their derivatives, and the moments $\left(M_{x}^{R_{b}}, M_{y}^{R_{b}}, M_{z}^{R_{b}}\right)$ applied to the aircraft originate from the general moment equation. $J$ is the inertia matrix and is the cross product.

$$
\left(\begin{array}{c}
\dot{p} \\
\dot{q} \\
\dot{r}
\end{array}\right)=J^{-1}\left[\left(\begin{array}{c}
M_{x}^{R_{b}} \\
M_{y}^{R_{b}} \\
M_{z}^{R_{b}}
\end{array}\right)-\left(\begin{array}{c}
p \\
q \\
r
\end{array}\right) \times J\left(\begin{array}{c}
p \\
q \\
r
\end{array}\right)\right] .
$$

The moments are expressed in $R_{b}$; they are due to aerodynamic effects and are modeled as follows:

$$
\left(\begin{array}{lll}
M_{x} & M_{y} & M_{z}
\end{array}\right)=\bar{q} S\left(\begin{array}{lll}
\bar{b} C_{l} & \bar{c} C_{m} & \bar{b} C_{n}
\end{array}\right) .
$$

Similarly, the coefficients of aerodynamic moment are denoted as follows.

$$
\begin{aligned}
C_{l}= & C_{l \beta} \beta+C_{l p} \frac{\bar{b} p}{2 V}+C_{l \delta_{\mathrm{ar}}} \delta_{\mathrm{ar}}+C_{l \delta_{\mathrm{al}}} \delta_{\mathrm{al}}+C_{l \delta_{\mathrm{fr}}} \delta_{\mathrm{fr}} \\
& +C_{l \delta_{\mathrm{fl}}} \delta_{\mathrm{fl}}+C_{l \delta_{\mathrm{er}}} \delta_{\mathrm{er}}+C_{l \delta_{\mathrm{el}}} \delta_{\mathrm{el}} \\
C_{m}= & C_{m 0}+C_{m \alpha} \alpha+C_{m q} \frac{\bar{c} q}{2 V}+C_{m \delta_{\mathrm{ar}}} \delta_{\mathrm{ar}}+C_{m \delta_{\mathrm{al}}} \delta_{\mathrm{al}} \\
& +C_{m \delta_{\mathrm{fr}}} \delta_{\mathrm{fr}}+C_{m \delta_{\mathrm{fl}}} \delta_{\mathrm{fl}}+C_{m \delta_{\mathrm{er}}} \delta_{\mathrm{er}}+C_{m \delta_{\mathrm{el}}} \delta_{\mathrm{el}} \\
C_{n}= & C_{n \beta} \beta+C_{n p} \frac{\bar{b} p}{2 V}+C_{n r} \frac{\bar{b} r}{2 V}+C_{n \delta_{\mathrm{ar}}} \delta_{\mathrm{ar}}+C_{n \delta_{\mathrm{al}}} \delta_{\mathrm{al}} \\
& +C_{n \delta_{\mathrm{fr}}} \delta_{\mathrm{fr}}+C_{n \delta_{\mathrm{f}}} \delta_{\mathrm{fl}}+C_{n \delta_{\mathrm{er}}} \delta_{\mathrm{er}}+C_{n \delta_{\mathrm{el}}} \delta_{\mathrm{el}} .
\end{aligned}
$$

From the above, the model of the flight control systems for $\mathrm{UAV}$, which is detailed in [4], is given by

$$
\begin{aligned}
& \dot{x}=f(x)+g(x) \delta, \\
& y=C x,
\end{aligned}
$$

where $x=[\phi, \theta, V, \alpha, \beta, p, q, r, h]^{T}$ is a state vector, which denotes roll angle, pitch angle, airspeed, attack angle, sideslip angle, roll rate, pitch rate, yaw rate, and altitude, respectively. $\delta=\left[\delta_{\mathrm{th}}, \delta_{\mathrm{ar}}, \delta_{\mathrm{al}}, \delta_{\mathrm{fr}}, \delta_{\mathrm{fl}}, \delta_{\mathrm{er}}, \delta_{\mathrm{el}}\right]^{T}$ is the control input vector, which includes the throttle, the right aileron, and left one, similarly, followed by flaps, the ruddervators ( $\mathrm{V}$ inverted tails). And the output vector is $y=[\phi, \theta, \beta, p, q, r]^{T}$ derived from various sensors onboard. To generalize the derivation, the above variables are denoted as $x \in R^{n}, \delta \in R^{m}, y \in R^{k}$.
2.2. Fuzzy T-S Model. The nonlinear system is linearized locally, which expresses the input-output relation of original system; then Takagi and Sugeno theory is used to establish the fuzzy dynamic model based on previous linearization [5]. The linear-fuzzy model can be represented as IF-THEN fuzzy rules; moreover, it can resolve relative control problem of FCS of UAV $[13,14]$. The model of the flight control systems for UAV could be expressed as a range of linearized models.

Consider a T-S fuzzy model which is composed of many fuzzy implications, where every implication is equal to a linear state-space model. The $i$ th fuzzy rule of the T-S model of FCS is written as follows.

Plant Rule $i$. IF $z_{1}(t)$ is $M_{i 1}$ and $z_{q}(t)$ is $M_{i q}$ THEN

$$
\begin{aligned}
& \dot{x}=A_{i} x+B_{i} \delta, \\
& y=C_{i} x,
\end{aligned}
$$

where $i=1 \cdots N$, the fuzzy rule number is defined as $N$, the fuzzy set is $M_{i j}(j=1 \cdots q), z(t)=\left[z_{1}(t) \cdots z_{q}(t)\right]^{T}$ are the given variable, $A_{i} x \in R^{n \times n}, B_{i} \in R^{n \times m}$, and $C_{i} \in R^{k \times n}$.

The overall fuzzy FCS for UAV with unknown disturbances $w$ could be deduced as follows:

$$
\begin{aligned}
& \dot{x}=\sum_{i=1}^{N} \pi_{i}(z)\left(A_{i} x+B_{i} \delta+B_{d} w\right), \\
& y=\sum_{i=1}^{N} \pi_{i}(z) C_{i} x,
\end{aligned}
$$

where $\pi_{i}(z)$ fulfils some constraint conditions as follows:

$$
\pi_{i}(z)=\frac{\prod_{j=1}^{q} M_{i j}(z)}{\sum_{i=1}^{N} \prod_{j=1}^{q} M_{i j}(z)},
$$

where $M_{i j}(z)$ is the grade of membership of $z_{j}(t)$ among $M_{i j}$. It is easily known that $\prod_{j=1}^{q} M_{i j}(z) \geq 0,0 \leq \pi_{i}(z) \leq 1$, and $\sum_{i=1}^{N} \pi_{i}(z)=1$ for all $t$ could be obtained.

Remark 1. The control input $\delta_{s}(s=1, \ldots, m)$, namely, output of controller, which is the deflection input of control surface, has to be continuous and bounded on physical meaning.

Since there are different mechanical and physical restrictions on the control surfaces or input amplitude, the output of actuator is denoted by the following $\operatorname{sat}(\delta)$ :

$$
\text { sat }\left(\delta_{s}\right)= \begin{cases}\delta_{s \min }, & \delta_{s}<\delta_{s \min } \\ \delta_{s}, & \delta_{s \min } \leq \delta_{s} \leq \delta_{s \max } \\ \delta_{s \max }, & \delta_{s}>\delta_{s \max },\end{cases}
$$

where $\operatorname{sat}(\delta)$ is the actual output of actuator with saturation constraints, $\delta_{\text {smin }}(s=1, \ldots, m)$ and $\delta_{s \max }(s=1, \ldots, m)$ are the minimum saturation level and maximum one of the 
output of actuator, which is decided in advance, and $\delta_{s}$ is the control input, namely, output of controller, that will be designed.

Obviously, the actual output of actuator energy provided may be smaller than the designed control input. In this sense, there is a difference between a real output of actuator provided and desired control input, described by

$$
\Delta \delta=\operatorname{sat}(\delta)-\delta
$$

where the saturation error produced by actuator saturation is expressed as $\Delta \delta$.

In an actual control system, the saturation error $\Delta \delta$ between an ideal input $\delta$ and a final output for actuator sat $(\delta)$ provided is bounded. Thus, it is assumed that the following inequality holds:

$$
\|\Delta \delta\| \leq h(x) \sigma^{*}
$$

where $h(x) \in R^{1 \times m}$ is a continuous known function and $\sigma^{*} \epsilon$ $R^{m \times 1}$ is an unknown parameter.

The overall fuzzy FCS for healthy UAV with unknown disturbances and actuator saturation are represented as follows:

$$
\begin{aligned}
& \dot{x}=\sum_{i=1}^{N} \pi_{i}(z)\left(A_{i} x+B_{i} \operatorname{sat}(\delta)+B_{d} w\right) \\
& y=\sum_{i=1}^{N} \pi_{i}(z) C_{i} x,
\end{aligned}
$$

where $\operatorname{sat}(\delta)$ defined as (11) denotes actual output of actuator of the healthy FCS and $\operatorname{sat}(\delta) \in R^{m}$.

Considering actuator faults, rewrite the fuzzy faulty FCS for UAV with unknown disturbances and actuator saturation as

$$
\begin{aligned}
& \dot{x}=\sum_{i=1}^{N} \pi_{i}(z)\left(A_{i} x+B_{i} \operatorname{sat}\left(\delta^{F}\right)+B_{d} w\right) \\
& y=\sum_{i=1}^{N} \pi_{i}(z) C_{i} x
\end{aligned}
$$

where $\delta^{F}$ is the input of a faulty FCS, $\operatorname{sat}\left(\delta^{F}\right)$ denotes an actual output of actuator of the faulty FCS, and $\delta^{F}=$ $\left[\delta_{1}^{F}, \delta_{2}^{F}, \ldots, \delta_{m}^{F}\right]^{T}$.

To formulate FTTC design, an actuator fault model has to be required as follows:

$$
\delta^{F}=\rho \delta
$$

In this paper, the actuator fault is set to be a loss of effectiveness (LOE) of control surface; $\rho \in R^{m \times m}$ is the unknown diagonal fault matrix with $\rho_{1}, \rho_{2}, \ldots, \rho_{m}$.

Furthermore, under actuator saturation condition, if $\delta_{s}^{F}$ is the $s_{\text {th }}$ faulty control input, then $\operatorname{sat}\left(\delta_{s}^{F}\right)$ represents the actual output of actuator from the $s_{\text {th }}$ actuator that has failed. Moreover, $\delta_{s}^{F}=\rho_{s} \delta_{s}$, where $\rho_{s}$ indicates a loss of effectiveness (LOE) element for $s_{\text {th }}$ actuator, $0<\rho_{s} \leq 1(s=1, \ldots, m)$.
From property of (12), the following equation is established:

$$
\begin{aligned}
\operatorname{sat}\left(\delta_{s}^{F}\right)=\delta_{s}^{F}+\Delta \delta_{s}^{F} & =\rho_{s} \delta_{s}+\Delta \delta_{s}^{F}, \\
& \rho_{s} \in\left[\underline{\rho_{s}}, \overline{\rho_{s}}\right], 0<\underline{\rho_{s}} \leq 1, \overline{\rho_{s}} \geq 1,
\end{aligned}
$$

where $\delta_{s}^{F}$ is control input and $\Delta \delta_{s}^{F}(s=1,2, \ldots, m)$ denotes a deviation between an actual output of actuator and ideal control input. $\rho_{s}$ is an unknown constant modeling $s_{\text {th }}$ control effectiveness element of $m$ control surfaces or actuators. $\overline{\rho_{s}}, \rho_{s}$ denote the known upper of $\rho_{s}$ bound and lower one, respectively.

It is worth noting that when $\rho_{s}=\overline{\rho_{s}}=1$, the $s_{\text {th }}$ actuator fault does not occur.

Between the minimum and maximum bounds $\left[\rho_{s}, \overline{\rho_{s}}\right]$, a set is defined as follows:

$$
\begin{aligned}
N_{\rho} & =\left\{\rho: \rho=\operatorname{diag}\left[\rho_{1}, \ldots, \rho_{m}\right], \rho_{s}=\underline{\rho_{s}} \text { or } \rho_{s}=\overline{\rho_{s}}, s\right. \\
& =1, \ldots, m\} .
\end{aligned}
$$

Remark 2. Here the loss of effectiveness fault is considered as control surface damage, so the saturation levels $\delta_{\text {smax }}, \delta_{\text {smin }}$ of the $s_{\text {th }}$ actuator are invariable whether or not the loss of effectiveness fault occurs, while actual output of actuator sat $(\delta)$ changes into sat $\left(\delta^{F}\right)$ due to $s_{\text {th }}$ actuator loss of effectiveness. Therefore, the following equation holds:

$$
\operatorname{sat}\left(\delta_{s}^{F}\right)= \begin{cases}\delta_{s \min }, & \delta_{s}^{F}<\delta_{s \min } \\ \delta_{s}^{F}, & \delta_{s \min } \leq \delta_{s}^{F} \leq \delta_{s \max } \\ \delta_{s \max }, & \delta_{s}^{F}>\delta_{s \max } .\end{cases}
$$

It is widely accepted that the steady-state tracking error is accommodated by an integral function of a controller. To design an adaptive controller with integral $\eta(t)=\int_{0}^{t}\left(y_{r}(s)-\right.$ $y(s)) d s$, combining (15) and $\eta(t)$, we obtain the following augmented system:

$$
\begin{aligned}
{\left[\begin{array}{l}
\dot{\eta} \\
\dot{x}
\end{array}\right] } & =\sum_{i=1}^{N} \pi_{i}(z) \\
& \cdot\left\{\left[\begin{array}{cc}
0 & -C_{i} \\
0 & A_{i}
\end{array}\right]\left[\begin{array}{l}
\eta \\
x
\end{array}\right]+\left[\begin{array}{l}
0 \\
B_{i}
\end{array}\right] \operatorname{sat}\left(\delta^{F}\right)+\left[\begin{array}{cc}
I & 0 \\
0 & B_{d}
\end{array}\right]\left[\begin{array}{l}
y_{r} \\
w
\end{array}\right]\right\} .
\end{aligned}
$$

Let $\bar{x}=\left[\begin{array}{ll}\eta^{T} & x^{T}\end{array}\right]^{T}$, and above fuzzy augmented system for UAV in faulty case can be changed into

$$
\dot{\bar{x}}=\sum_{i=1}^{N} \pi_{i}(z)\left(\mathbb{A}_{i} \bar{x}+\mathbb{B}_{i} \operatorname{sat}\left(\delta^{F}\right)\right)+\mathbb{B}_{d} d,
$$


where

$$
\begin{aligned}
\mathbb{A}_{i} & =\left[\begin{array}{ll}
0 & -C_{i} \\
0 & A_{i}
\end{array}\right], \\
\mathbb{B}_{i} & =\left[\begin{array}{l}
0 \\
B_{i}
\end{array}\right], \\
\mathbb{B}_{d} & =\left[\begin{array}{ll}
I & 0 \\
0 & B_{d}
\end{array}\right], \\
d & =\left[\begin{array}{l}
y_{r} \\
w
\end{array}\right] .
\end{aligned}
$$

Note that if the failure parameter $\rho=I$ ( $I$ is an identity matrix), then no fault happens and $\operatorname{sat}(\delta)=\operatorname{sat}\left(\delta^{F}\right)$.

So the fuzzy augmented system for UAV in fault free case is written by

$$
\dot{\bar{x}}=\sum_{i=1}^{N} \pi_{i}(z)\left(\mathbb{A}_{i} \bar{x}+\mathbb{B}_{i} \text { sat }(\delta)\right)+\mathbb{B}_{d} d .
$$

The design of an adaptive fault tolerant controller is final target, which is accomplished to track an ideal output $y_{r}$ of a reference system when unknown disturbances, actuator saturation, and faults occur and guarantee the given dynamic performance. All the output of FTC system is asymptotically convergent to the ideal output of flight control systems $y_{r}$ by the developed controller.

For the faulty system described by (21) and healthy system (23), the problem becomes finding a FTTC such that the following conditions exist.

(i) During normal operation, the controlled system in fault free case is stable; moreover, our required output $y$ tracks the reference command $y_{r} \in R^{k}$ under the condition of no steady-state error; namely,

$$
\begin{aligned}
\lim _{t \rightarrow \infty} \varepsilon & =0, \\
\varepsilon & =y_{r}-y, \\
\eta & =\int_{0}^{t} \varepsilon(s) d s,
\end{aligned}
$$

where $y_{r}$ is the output command, which is given by a reference model.

Besides, the robust tracking control performance index $\gamma$ for all $d(t)$ is satisfied:

$$
\int_{0}^{t} \eta^{T}(s) \eta(s) d s \leq \gamma^{2} \int_{0}^{t} d^{T}(s) d(s) d s .
$$

(ii) During fault tolerant control operation, the controlled system with actuator faults is stable; in addition a required output $y$ can still track the trajectory $y_{r}$ and no steady-state error.

To proceed with the design of robust FTC for a faulty UAV with disturbances and saturation constraints, three assumptions, in turn, are as follows.
Assumption 3. The loss of effectiveness (LOE) of the actuator is bounded; moreover, there exists a positive scalar $\omega>0$ such that $\omega \leq\|\rho\| \leq 1$ holds.

Assumption 4. The unknown disturbance $w$ is bounded; namely, there exists a positive scalar $\varsigma>0$ such that $\|w\|<\varsigma$ holds.

\section{Main Results}

In the following section, the novel FTTC scheme is designed using the adaptive control theory to accommodate the function of saturation and faults with no need for any diagnosis unit; moreover the previous required control objective would be implemented.

Considering the T-S fuzzy system (21) with saturation constraints and faults, an appropriate adaptive FTC scheme is developed to meet the following two requirements. In the normal case, there are no actuator faults, but actuator saturation constraints and disturbances, if the developed controller guarantees the asymptotic stability of system, and the output $y$ will be required to track the given trajectory $y_{r}$ asymptotically. In actuator faulty case, the developed FTTC guarantees the asymptotic stability and tracking performance of system similarly.

3.1. Normal Control Law Design for an UAV with Input Saturation. When there is no fault in the flight control systems for UAV (23), one considers the following controller including $H_{\infty}$ state-feedback control:

$$
\begin{aligned}
\delta & =\sum_{j=1}^{N} \pi_{j}(z) \mathbb{K}_{j} \bar{x}+u_{2} \\
& =\sum_{j=1}^{N} \pi_{j}(z)\left[\begin{array}{ll}
K_{j \eta} & K_{j x}
\end{array}\right]\left[\begin{array}{ll}
\eta^{T} & x^{T}
\end{array}\right]^{T}+u_{2},
\end{aligned}
$$

where the normal control input is $\delta$, the feedback gain matrix is $\mathbb{K}_{j} \in R^{m \times(k+n)}$ to be determined and $\mathbb{K}_{j}=\left[K_{j \eta} K_{j x}\right]$, and the adaptive control input is $u_{2} \in R^{m}$ used to compensate for the actuator saturation.

Substituting (26) into (23), the controlled fuzzy system is described as

$$
\begin{aligned}
\dot{\bar{x}}= & \sum_{i=1}^{N} \sum_{j=1}^{N} \pi_{i}(z) \pi_{j}(z)\left(\mathbb{A}_{i}+\mathbb{B}_{i} \mathbb{K}_{j}\right) \bar{x} \\
& +\sum_{i=1}^{N} \pi_{i}(z)\left[\mathbb{B}_{i} \Delta \delta+\mathbb{B}_{i} u_{2}\right]+\mathbb{B}_{d} d .
\end{aligned}
$$

Next, $u_{2}$ is designed for the asymptotic stability of output tracking error.

The Lyapunov function candidate is defined.

$$
V=\bar{x}^{T} P \bar{x}+\sum_{s=1}^{m} \frac{\widetilde{\sigma}_{s}^{2}}{\vartheta_{s}},
$$


where $\widehat{\sigma}$ is the estimation value of $\sigma^{*}, \sigma^{*}=\left[\sigma_{1}, \ldots, \sigma_{m}\right]^{T}$, and $\widetilde{\sigma}=\sigma^{*}-\widehat{\sigma} \cdot P=P^{T}>0$ and $\vartheta_{s}>0$ is a learning coefficient determined by $\sigma_{s}, s=1, \ldots, m$.

The time derivative of $V$ can be written as

$$
\dot{V}=2 \bar{x}^{T} P \dot{\bar{x}}+2 \sum_{s=1}^{m} \frac{\widetilde{\sigma}_{s}^{T} \dot{\hat{\sigma}}_{s}}{\vartheta_{s}} .
$$

Invoking (27) and (29), one obtains

$$
\begin{aligned}
\dot{V} & =\sum_{i=1}^{N} \sum_{j=1}^{N} \pi_{i}(z) \pi_{j}(z) \\
& \cdot\left[\bar{x}^{T} P\left(\mathbb{A}_{i}+\mathbb{B}_{i} \mathbb{K}_{j}\right)+\left(\mathbb{A}_{i}+\mathbb{B}_{i} \mathbb{K}_{j}\right)^{T} P \bar{x}\right] \\
& +2 \sum_{i=1}^{N} \pi_{i}(z)\left[\bar{x}^{T} P \mathbb{B}_{i} \Delta \delta+\bar{x}^{T} P \mathbb{B}_{i} u_{2}\right]+2 \sum_{s=1}^{m} \frac{\widetilde{\sigma}_{s}^{T} \dot{\hat{\sigma}}_{s}}{\vartheta_{s}} \\
& +2 \bar{x}^{T} P \mathbb{B}_{d} d .
\end{aligned}
$$

For (13), (30) can be rewritten by

$$
\begin{aligned}
\dot{V} & \leq \sum_{i=1}^{N} \sum_{j=1}^{N} \pi_{i}(z) \pi_{j}(z) \\
\cdot & {\left[\bar{x}^{T}\left(P\left(\mathbb{A}_{i}+\mathbb{B}_{i} \mathbb{K}_{j}\right)+\left(\mathbb{A}_{i}+\mathbb{B}_{i} \mathbb{K}_{j}\right)^{T} P\right) \bar{x}\right] } \\
& +2 \sum_{i=1}^{N} \pi_{i}(z)\left[\left\|\bar{x}^{T} P \mathbb{B}_{i}\right\| h(x) \sigma^{*}+\bar{x}^{T} P \mathbb{B}_{i} u_{2}\right] \\
& +2 \sum_{s=1}^{m} \frac{\widetilde{\sigma}_{s}^{T} \dot{\hat{\sigma}}_{s}}{\vartheta_{s}}+2 \bar{x}^{T} P \mathbb{B}_{d} d .
\end{aligned}
$$

Choose the following compensate control law:

$$
u_{2}(t)=-\frac{\mathbb{B}_{i}^{T} P \bar{x}(t)}{\left\|\bar{x}^{T}(t) P \mathbb{B}_{i}\right\|} h(x) \widehat{\sigma},
$$

where $\widehat{\sigma}$ is an estimation of $\sigma^{*}$.

Invoking (32), (31) is deduced to

$$
\begin{aligned}
\dot{V} & \leq \sum_{i=1}^{N} \sum_{j=1}^{N} \pi_{i}(z) \pi_{j}(z) \\
\cdot & {\left[\bar{x}^{T}\left(P\left(\mathbb{A}_{i}+\mathbb{B}_{i} \mathbb{K}_{j}\right)+\left(\mathbb{A}_{i}+\mathbb{B}_{i} \mathbb{K}_{j}\right)^{T} P\right) \bar{x}\right] } \\
& +2 \sum_{i=1}^{N} \pi_{i}(z)\left[\left\|\bar{x}^{T} P \mathbb{B}_{i}\right\| h(x) \tilde{\sigma}\right]+2 \sum_{s=1}^{m} \frac{\widetilde{\sigma}_{s}^{T} \dot{\hat{\sigma}}_{s}}{\vartheta_{s}} \\
& +2 \bar{x}^{T} P \mathbb{B}_{d} d .
\end{aligned}
$$

The parameter estimation law of $\widehat{\sigma}$ is presented as

$$
\dot{\hat{\sigma}}_{s}=-\sum_{i=1}^{N} \pi_{i}(z) \vartheta_{s} h_{s}^{T}(x)\left\|\bar{x}^{T} P \mathbb{B}_{i}\right\|,
$$

where $h=\left[h_{1}, \ldots, h_{m}\right]$ and $s=1, \ldots, m$.
Substituting (34) into (33), it follows that

$$
\begin{aligned}
\dot{V} & \leq \sum_{i=1}^{N} \sum_{j=1}^{N} \pi_{i}(z) \pi_{j}(z) \\
& \cdot\left[\bar{x}^{T}\left(P\left(\mathbb{A}_{i}+\mathbb{B}_{i} \mathbb{K}_{j}\right)+\left(\mathbb{A}_{i}+\mathbb{B}_{i} \mathbb{K}_{j}\right)^{T} P\right) \bar{x}\right] \\
& +2 \bar{x}^{T} P \mathbb{B}_{d} d .
\end{aligned}
$$

Theorem 5. If there exist a set of real matrices $\mathbb{Z}_{j} \in R^{m \times(k+n)}$ and a symmetric matrix $\mathbb{R} \in R^{(n+k) \times(k+n)}$, for a given positive scalar $\gamma>0$, such that inequalities (36) hold,

$$
\begin{aligned}
L_{i i}<0, \quad 1 \leq i \neq j \leq N & \\
\frac{2}{N-1} L_{i i}+L_{i j}+L_{j i} & <0,
\end{aligned}
$$

where

$$
L_{i j}=\left[\begin{array}{ccc}
\mathbb{A}_{i} \mathbb{R}+\mathbb{B}_{i} \mathbb{Z}_{j}+\left(\mathbb{A}_{i} \mathbb{R}+\mathbb{B}_{i} \mathbb{Z}_{j}\right)^{T} & \mathbb{B}_{d} & \mathbb{R} \\
\mathbb{B}_{d}^{T} & -\gamma^{2} I & 0 \\
\mathbb{R} & 0 & -I
\end{array}\right]
$$

then a healthy T-S fuzzy FCS (23) is asymptotic stable using the following adaptive controller:

$$
\begin{aligned}
& \delta=\sum_{j=1}^{N} \pi_{j}(z) \mathbb{K}_{j} \bar{x}-\frac{\mathbb{B}_{i}^{T} P \bar{x}}{\left\|\bar{x}^{T} P \mathbb{B}_{i}\right\|} h(x) \widehat{\sigma} \\
& \mathbb{K}_{j}=\mathbb{Z}_{j} \mathbb{R}^{-1}, j=1, \ldots, N .
\end{aligned}
$$

Proof. If the FCS (23) is stable, it is known that

$$
\dot{V}<0 .
$$

Moreover, the performance of control system with attenuation level $\gamma$ for all $d$ could be guaranteed if next condition is fulfilled.

$$
\int x^{T} x d t \leq \gamma^{2} \int d^{T} d d t
$$

The condition described in (25) is the $H_{\infty}$ performance index $\gamma$ condition satisfied for all $d$. And the FCS with $H_{\infty}$ performance index $\gamma$ is guaranteed if the following inequality holds:

$$
\int \bar{x}^{T} \bar{x} d t \leq \gamma^{2} \int d^{T} d d t
$$

where the definition of $\bar{x}$ is the same as (20). 
So the stability of FCS (23) with $H_{\infty}$ performance index $\gamma$ could be guaranteed if condition (42) is fulfilled.

$$
\dot{V}+\bar{x}^{T} \bar{x}-\gamma^{2} d^{T} d<0
$$

which leads to

$$
\begin{aligned}
& \sum_{j=1}^{N} \mu_{i}(z) \mu_{j}(z) \\
& \cdot \bar{x}^{T}\left[P\left(\mathbb{A}_{i}+\mathbb{B}_{i} \mathbb{K}_{j}\right)+\left(\mathbb{A}_{i}+\mathbb{B}_{i} \mathbb{K}_{j}\right)^{T} P+I\right] \bar{x} \\
& \quad+\bar{x}^{T} P \mathbb{B}_{d} d+d^{T} \mathbb{B}_{d}^{T} P \bar{x}-\gamma^{2} d^{T} d<0 .
\end{aligned}
$$

The formula above is also equivalent to

$$
\begin{aligned}
& \sum_{j=1}^{N} \pi_{i}(z) \pi_{j}(z)\left[\begin{array}{l}
\bar{x} \\
d
\end{array}\right]^{T} \\
& \quad \cdot\left[\begin{array}{cc}
P\left(\mathbb{A}_{i}+\mathbb{B}_{i} \mathbb{K}_{j}\right)+\left(\mathbb{A}_{i}+\mathbb{B}_{i} \mathbb{K}_{j}\right)^{T} P+I & P \mathbb{B}_{d} \\
\mathbb{B}_{d}^{T} P & -\gamma^{2} I
\end{array}\right]\left[\begin{array}{l}
\bar{x} \\
d
\end{array}\right] \\
& \quad<0 .
\end{aligned}
$$

Let $P^{-1}=\mathbb{R}$; after two primary transforms, inequality (44) changes into

$$
\begin{aligned}
& {\left[\begin{array}{cc}
\left(\mathbb{A}_{i}+\mathbb{B}_{i} \mathbb{K}_{j}\right) \mathbb{R}+\mathbb{R}^{T}\left(\mathbb{A}_{i}+\mathbb{B}_{i} \mathbb{K}_{j}\right)^{T}+\mathbb{R}^{T} \mathbb{R} & \mathbb{B}_{d} \\
\mathbb{B}_{d}^{T} & -\gamma^{2} I
\end{array}\right]} \\
& <0
\end{aligned}
$$

which, considering Schur complement, can be implemented, if the inequalities (36) hold [15]. From the preceding deduction, inequality (45) holds, and the asymptotical stability of FCS (23) with $\gamma$-disturbance attenuation level is accomplished. The proof is completed.

The results of the above theorem are suitable for system (23) in fault free case. When certain control surfaces lose partial control effectiveness, an adaptive normal controller proposed in (26) will not implement the required tracking control objective so we have to develop a new FTTC.

3.2. Adaptive FTTC Design. Here, a FTC law $\delta^{F}$ utilizing adaptive fault compensation controller is proposed

$$
\delta^{F}=\delta+\delta^{C}
$$

where $\delta$ is the normal control input term presented in (26) and $\delta^{C}$ is an adaptive fault compensation factor so whether it is zero or not lies on whether actuator faults occur.

As shown in Figure 2, the configuration of the adaptive FTC scheme is addressed in our paper.

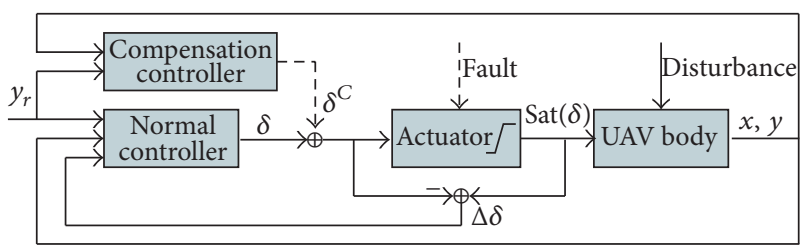

FIgURE 2: The adaptive fault tolerant control system structure.

Next, to acquire a necessary estimation of actuator faults, a target model is given:

$$
\begin{aligned}
& \dot{\hat{x}}=\sum_{i=1}^{N} \pi_{i}(z)\left[A_{i} \widehat{x}+B_{i} \hat{\rho} r+B_{i} u_{3}\right] \\
& \widehat{y}=\sum_{i=1}^{N} \pi_{i}(z) C_{i} \widehat{x},
\end{aligned}
$$

where $\hat{\rho}=\operatorname{diag}\left[\hat{\rho}_{1}, \ldots, \hat{\rho}_{m}\right]$ expresses the estimation of remaining effectiveness factor. To implement given control objective, the inputs $r \in R^{m}$ and $u_{3} \in R^{m}$ are determined later.

To design the suitable input $\delta^{F}$, the output $y$ of system (21) with actuator saturation (13), unknown disturbances, and actuator faults (16) can track the trajectory $y_{r}$ asymptotically; the augmented target model can be introduced

$$
\begin{aligned}
{\left[\begin{array}{c}
\dot{\hat{\eta}} \\
\dot{\hat{x}}
\end{array}\right] } & =\sum_{i=1}^{N} \pi_{i}(z) \\
& \cdot\left\{\left[\begin{array}{cc}
0 & -C_{i} \\
0 & A_{i}
\end{array}\right]\left[\begin{array}{l}
\hat{\eta} \\
\hat{x}
\end{array}\right]+\left[\begin{array}{l}
0 \\
B_{i}
\end{array}\right] \widehat{\rho} r+\left[\begin{array}{l}
0 \\
B_{i}
\end{array}\right] u_{3}+\left[\begin{array}{ll}
I & 0 \\
0 & 0
\end{array}\right]\left[\begin{array}{l}
y_{r} \\
w
\end{array}\right]\right\} .
\end{aligned}
$$

which is expressed as follows:

$$
\dot{\overline{\bar{x}}}=\sum_{i=1}^{N} \pi_{i}(z)\left[\mathbb{A}_{i} \hat{\bar{x}}+\mathbb{B}_{i}\left(\hat{\rho} r+u_{3}\right)\right]+\mathbb{D}_{1} d,
$$

where $\widehat{\bar{x}}=\left[\widehat{\eta}^{T}, \widehat{x}^{T}\right]^{T}, \mathbb{A}_{i}, \mathbb{B}_{i}$, and $d$ are the same as those in normal operation (24), and

$$
\mathbb{D}_{1}=\left[\begin{array}{ll}
I & 0 \\
0 & 0
\end{array}\right] .
$$

If one defines the state error vector of the augmented system as $e=\bar{x}-\widehat{\bar{x}}$ and assumes a fault tolerant control law

$$
\delta^{F}=r+\sum_{j=1}^{N} \mathbb{F}_{j} e
$$

and a dynamics including (21) and (48) is deduced as

$$
\begin{aligned}
\dot{e}= & \sum_{i=1}^{N} \sum_{j=1}^{N} \pi_{i}(z) \pi_{j}(z) \\
& \cdot\left[\left(\mathbb{A}_{i}+\mathbb{B}_{i} \rho \mathbb{F}_{j}\right) e+\mathbb{B}_{i} \widetilde{\rho} r+\mathbb{B}_{i}\left(\Delta \delta^{F}-u_{3}\right)+\widetilde{\mathbb{B}}_{d} d\right],
\end{aligned}
$$


where $\tilde{\rho}_{s}=\rho_{s}-\hat{\rho}_{s}(s=1,2, \ldots, m), \widetilde{\mathbb{B}}_{d}=\mathbb{B}_{d}-\mathbb{D}_{1}$, and $\mathbb{F}_{j}(j=1, \ldots, m)$ is a difference control gain, which is designed to stabilize a T-S fuzzy system (52).

Let $r=\left[r_{1}, \ldots, r_{m}\right]^{T}$ and $\mathbb{B}_{i}=\left[b_{i 1}, \ldots, b_{i m}\right]$; the augmented system (52) is described by

$$
\begin{aligned}
\dot{e}= & \sum_{i=1}^{N} \pi_{i}(z)\left[\sum_{j=1}^{N} \pi_{j}(z)\left(\mathbb{A}_{i}+\mathbb{B}_{i} \rho \mathbb{F}_{j}\right) e+\sum_{s=1}^{m} b_{i s} \tilde{\rho}_{s} r_{s}\right. \\
& \left.+\mathbb{B}_{i}\left(\Delta \delta^{F}-u_{3}\right)\right]+\widetilde{\mathbb{B}}_{d} d .
\end{aligned}
$$

Theorem 6. The augmented T-S fuzzy system (53) with $\gamma$ disturbance attenuation is asymptotically stable, as long as there exist real matrices $\mathbb{W} \in R^{m \times(n+k)}, \mathbb{W}_{j}>0, j=1, \ldots, N$,

$$
\dot{\hat{\rho}}_{s}=\operatorname{Proj}_{\left[\underline{\left.\rho_{s}, \bar{\rho}_{s}\right]}\right.}\left\{l_{s} e^{T} G b_{i s} r_{s}\right\}=\left\{\begin{array}{l}
0, \\
\sum_{i=1}^{N} \pi_{i}(z) l_{s} e^{T} G b_{i s} r_{s},
\end{array}\right.
$$

if $\hat{\rho}_{s}=\overline{\rho_{s}}, l_{s} e^{T} G b_{i s} r_{s} \geq 0$ or $\hat{\rho}_{s}=\underline{\rho_{s}}, l_{s} e^{T} G b_{i s} r_{s} \leq 0$

where $l_{s}>0$ denotes a learning parameter to be designed by the minimum bound of fault and maximum one $\left(\rho_{s}, \overline{\rho_{s}}\right)$, the projection operator is expressed by Proj $\{\cdot\}$, which is to project an estimation $\hat{\rho}_{s}$ in the range $\left[\rho_{s}, \overline{\rho_{s}}\right]$, and an error control gain $\mathbb{F}_{j}$ is deduced by $\mathbb{F}_{j}=\mathbb{W}_{j} \mathbb{Q}^{-1}$.

Proof. Let Lyapunov function be

$$
V_{1}=e^{T} G e+\sum_{s=1}^{m} \frac{\tilde{\rho}_{s}^{2}}{l_{s}}
$$

where $G=\mathbb{Q}^{-1}>0$.

Along the trajectory of the augmented system (53), a derivative of $V_{1}$ can be deduced as

$$
\begin{aligned}
\dot{V}_{1} & =\sum_{i=1}^{N} \sum_{j=1}^{N} \pi_{i}(z) \pi_{j}(z) \\
\cdot & e^{T}\left[G\left(\mathbb{A}_{i}+\mathbb{B}_{i} \rho \mathbb{F}_{j}\right)+\left(\mathbb{A}_{i}+\mathbb{B}_{i} \rho \mathbb{F}_{j}\right)^{T} G\right] e \\
& +e^{T} G \mathbb{B}_{d} d+d^{T} \mathbb{B}_{d}^{T} G e+2 \sum_{i=1}^{N} \pi_{i}(z) e^{T} G \mathbb{B}_{i}\left(\Delta \delta^{F}\right. \\
& \left.-u_{3}\right)+2 \sum_{s=1}^{m} \sum_{i=1}^{N} \pi_{i}(z) \tilde{\rho}_{s} e^{T} G b_{i s} r_{s}+2 \sum_{s=1}^{m} \frac{\tilde{\rho}_{s}}{l_{s}}
\end{aligned}
$$

Considering that $\rho_{s}$ is an unknown scalar, one can easily obtain that $\dot{\tilde{\rho}}_{s}=-\dot{\hat{\rho}}_{s}$. otherwise,

and symmetric positive matrices $\mathbb{Q} \in R^{(n+k) \times(n+k)}$ such that two conditions hold as follows:

$$
\begin{aligned}
H_{i i} & <0, \quad 1 \leq i \neq j \leq N \\
\frac{2}{N-1} H_{i i}+H_{i j}+H_{j i} & <0,
\end{aligned}
$$

with

$$
\begin{aligned}
& H_{i j} \\
& \quad=\left[\begin{array}{ccc}
\mathbb{A}_{i} \mathbb{Q}+\mathbb{B}_{i} \rho \mathbb{W}_{j}+\left(\mathbb{A}_{i} \mathbb{Q}+\mathbb{B}_{i} \rho \mathbb{W}_{j}\right)^{T} & \mathbb{B}_{d} & \mathbb{Q} \\
\mathbb{B}_{d}^{T} & -\gamma^{2} I & 0 \\
\mathbb{Q} & 0 & -I
\end{array}\right]
\end{aligned}
$$

and $\hat{\rho}_{s},(s=1, \ldots, m)$ are determined on the basis of an

Based on (56), one has

$$
\frac{\tilde{\rho}_{s} \dot{\hat{\rho}}_{s}}{l_{s}}=\sum_{i=1}^{m} \pi_{i}(z) \tilde{\rho}_{s} e^{T} G b_{i s} r_{s} .
$$

From (59), one obtains the following equality:

$$
\begin{aligned}
\dot{V}_{1} & =\sum_{i=1}^{N} \sum_{j=1}^{N} \pi_{i}(z) \pi_{j}(z) \\
& \cdot e^{T}\left[G\left(\mathbb{A}_{i}+\mathbb{B}_{i} \rho \mathbb{F}_{j}\right)+\left(\mathbb{A}_{i}+\mathbb{B}_{i} \rho \mathbb{F}_{j}\right)^{T} G\right] e \\
& +2 \sum_{i=1}^{N} \pi_{i}(z) e^{T} G \mathbb{B}_{i}\left(\Delta \delta^{F}-u_{3}\right)+e^{T} G \mathbb{B}_{d} d \\
& +d^{T} \mathbb{B}_{d}^{T} G e .
\end{aligned}
$$

Considering Remark $1,\left\|\Delta \delta^{F}\right\|$ is bounded and not larger than $h(x) \xi$, where $\xi \in R^{m \times 1}$ is the unknown vector, $\widehat{\xi}$ is the estimated value for $\xi$, and $h(x)$ is defined as (13).

The control term $u_{3}$ is a compensation controller to accommodate the effect of actuator saturation, which is given by

$$
u_{3}=-\frac{\mathbb{B}_{i}^{T} G e}{\left\|e^{T} G \mathbb{B}_{i}\right\|} h(x) \hat{\xi},
$$

where $\hat{\xi}$ is the estimate of the unknown vector $\xi$. Similar to Remark 7, when $u_{3}=0, \widehat{\xi}=0$ and $\Delta \delta^{F}=0$ are acquired. adaptive estimation algorithm as follows: 
Invoking (61), (60) is described by

$$
\begin{aligned}
\dot{V}_{1} & \leq \sum_{i=1}^{N} \sum_{j=1}^{N} \pi_{i}(z) \pi_{j}(z) \\
\cdot & e^{T}\left[G\left(\mathbb{A}_{i}+\mathbb{B}_{i} \rho \mathbb{F}_{j}\right)+\left(\mathbb{A}_{i}+\mathbb{B}_{i} \rho \mathbb{F}_{j}\right)^{\mathrm{T}} G\right] e \\
& +2 \sum_{i=1}^{N} \pi_{i}(z)\left\|e^{T} G \mathbb{B}_{i}\right\| h(x) \tilde{\xi}+e^{T} G \mathbb{B}_{d} d \\
& +d^{T} \mathbb{B}_{d}^{T} G e,
\end{aligned}
$$

where $\widetilde{\xi}=\xi-\widehat{\xi}$.

Lyapunov function candidate is selected:

$$
V_{2}(t)=\sum_{s=1}^{m} \frac{\widetilde{\xi}_{s}^{T} \widetilde{\xi}_{s}}{\zeta_{s}}
$$

where $\widetilde{\xi}_{s}=\xi_{s}-\widehat{\xi}_{s}(s=1, \ldots, m) . \zeta_{s}>0$ is an adaptive gain determined by $\xi_{s}$.

Since $\xi$ is an unknown constant vector, the below equation is obtained:

$$
\dot{V}_{2}=2 \sum_{s=1}^{m} \frac{\tilde{\xi}_{s}^{T} \dot{\hat{\xi}}_{s}}{\zeta_{s}}
$$

Choosing the adaptive parameter update law

$$
\dot{\vec{\xi}}_{s}=-\sum_{i=1}^{N} \pi_{i}(z) \zeta_{s}\left\|e^{T} G \mathbb{B}_{i}\right\| h_{s}(x),
$$

where $h=\left[h_{1}, \ldots, h_{m}\right]$ and $s=1, \ldots, m$.

And let

$$
V=V_{1}+V_{2}
$$

Then

$$
\begin{aligned}
\dot{V} & \leq \sum_{i=1}^{N} \sum_{j=1}^{N} \pi_{i}(z) \pi_{j}(z) \\
& \cdot e^{T}\left[G\left(\mathbb{A}_{i}+\mathbb{B}_{i} \rho \mathbb{F}_{j}\right)\left(\mathbb{A}_{i}+\mathbb{B}_{i} \rho \mathbb{F}_{j}\right)^{T} G\right] e \\
& +e^{T} G \mathbb{B}_{d} d+d^{T} \mathbb{B}_{d}^{T} G e .
\end{aligned}
$$

Under zero initial condition, if condition (68) holds, the stability of (53) with $H_{\infty}$ performance index $\gamma$ can be guaranteed:

$$
\begin{aligned}
& \dot{V}(t)+e^{T} e-\gamma^{2} d^{T} d=\sum_{i=1}^{N} \sum_{j=1}^{N} \pi_{i}(z) \pi_{j}(z) . \\
& {\left[\begin{array}{l}
e \\
d
\end{array}\right]^{T}} \\
& \quad\left[\begin{array}{cc}
G\left(\mathbb{A}_{i}+\mathbb{B}_{i} \rho \mathbb{F}_{j}\right)+\left(\mathbb{A}_{i}+\mathbb{B}_{i} \rho \mathbb{F}_{j}\right)^{T} G+I & G \widetilde{B}_{d} \\
\widetilde{B}_{d}^{T} G & -\gamma^{2} I
\end{array}\right]\left[\begin{array}{l}
e \\
d
\end{array}\right] \\
& <0
\end{aligned}
$$

If $\mathbb{F}_{j}=\mathbb{W}_{j} \mathbb{Q}^{-1}, \mathbb{Q}=G^{-1},(68)$ is equivalent to the following:

$$
\begin{aligned}
& {\left[\begin{array}{cc}
\left(\mathbb{A}_{i}+\mathbb{B}_{i} \rho \mathbb{F}_{j}\right) \mathbb{Q}+\mathbb{Q}^{T}\left(\mathbb{A}_{i}+\mathbb{B}_{i} \rho \mathbb{F}_{j}\right)^{T}+\mathbb{Q}^{T} \mathbb{Q} & \widetilde{B}_{d} \\
\widetilde{B}_{d}^{T} & -\gamma^{2} I
\end{array}\right]} \\
& <0 \text {. }
\end{aligned}
$$

In addition, the following inequality could be derived for $\forall \rho \in N_{\rho}$

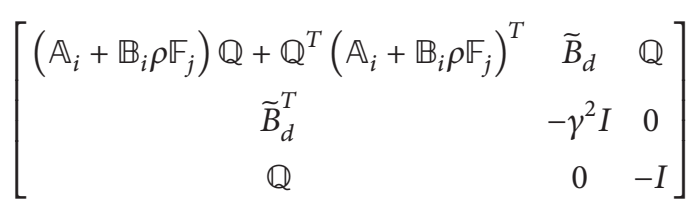

$<0$.

The proof is completed. For Lyapunov stability theory, when actuator faults in (16) and actuator saturation of (11) occur, it is obvious from (68) that the augmented T-S fuzzy system (53) with disturbance attenuation level $\gamma$ is asymptotically stable using the adaptive estimation law (56).

Then, a new adaptive FTC algorithm could be obtained

$$
\begin{aligned}
\delta^{F} & =r+\sum_{j=1}^{N} \pi_{j}(z) \mathbb{F}_{j} e \\
& =\sum_{j=1}^{N} \pi_{j}(z)\left[\widehat{\rho}^{-1} \mathbb{K}_{j} \hat{x}+\mathbb{F}_{j} e\right]-\frac{\mathbb{B}_{i}^{T} P \bar{x}}{\left\|\bar{x}^{T} P \mathbb{B}_{i}\right\|} h(x) \widehat{\sigma} \\
& =\delta+\delta^{C},
\end{aligned}
$$

where $\delta=\sum_{j=1}^{N} \pi_{j}(z) \mathbb{K}_{j} \bar{x}-\left(\mathbb{B}_{i}^{T} P \bar{x} /\left\|\bar{x}^{T} P \mathbb{B}_{i}\right\|\right) h(x) \widehat{\sigma}$ and $\delta^{C}=$ $\sum_{j=1}^{N} \pi_{j}(z)\left[\hat{\rho}^{-1}(I-\hat{\rho}) \mathbb{K}_{j} \hat{\bar{x}}+\left(\mathbb{F}_{j}-\mathbb{K}_{j}\right) e\right]$.

If control input does not exceed the saturation level of the actuator, namely, $\Delta \delta=0$, then the second adaptive term compensating the actuator saturation converges to zero and $\delta$ would be a normal state-feedback controller. The FTC approach of T-S fuzzy system presented in this paper does not depend on the fault diagnosis unit, which could reduce the complexity of controller design and be easily applied on nonlinear FCS of UAV.

Remark 7. Our research is motivated by some results in [28]. There are three main differences compared with the approach obtained in [28]. Firstly, the controlled system addressed in [28] is a linear control system without actuator saturation, while the nonlinear FCS with actuator saturation are investigated in our paper. Secondly, authors of [28] have not studied influence of unknown disturbance while we consider it. Thirdly, the scheme of [28] just relies on adaptive control, while our design discussed about this research combines adaptive control and $H$-infinity performance. So our results of nonlinear flight control systems for UAV under unknown disturbance, actuator LOE faults, and saturation are deemed to be complements or extension for [28]. 


\section{Illustrative Example}

In the following simulation, the efficiency of the presented controller is demonstrated. The nonlinear flight control systems for UAV refers to [4], where $x=[\phi, \theta, V, \alpha, \beta, p, q, r, h]^{T}$ is the state vector, $\delta=\left[\delta_{\mathrm{th}}, \delta_{\mathrm{ar}}, \delta_{\mathrm{al}}, \delta_{\mathrm{fr}}, \delta_{\mathrm{fl}}, \delta_{\mathrm{er}}, \delta_{\mathrm{el}}\right]^{T}$ is the input vector, and $y=[\phi, \theta, \beta, p, q, r]^{T}$ expresses the output vector. $w$ represents the unknown disturbance and $\rho=\operatorname{diag}\left[\rho_{1}, \ldots, \rho_{7}\right]$ is the unknown scalar modeling the remaining control effectiveness of seven actuator channels.

Here the unit of degree is expressed as (deg), unit of angular rate is (deg/s), airspeed unit is $(\mathrm{m} / \mathrm{s})$, and altitude unit is $(\mathrm{m})$.

Consider that the nonlinearity of UAV flight control systems mainly comes from airspeed $V$ and altitude $h$. In the airspeed range $V \in[15,50]$, we assume that $V$ has two related fuzzy sets $\{V=15\}$ and $\{V=50\}$ and $h$ has three related fuzzy sets $\{h=200\},\{h=1500\}$, and $\{h=$ 3000\}. The similar corresponding membership functions are obtained as [16]. We choose six operating points: $[V, h] \epsilon$ $\{[15,200],[15,1500],[15,3000],[50,200],[50,1500]$, and $[50,3000]\}$. Under the membership functions and the six operating points, six plant rules and six control rules can be defined. All $A_{i}$ and $B_{i}$ can be obtained by substituting the six operating points to $f(x), g(x)$.

Here, each of the seven actuators may lose its effectiveness and $\rho_{s} \in(0,1], s=1,2, \ldots, 7$. The scope of every effectiveness element is $\underline{\rho_{s}} \in(0,1], \overline{\rho_{s}} \in[1, \infty)(s=1,2, \ldots, 7)$, respectively. The tracking command of $\phi, \theta, \beta, p, q, r$ is

$$
K_{1 x}=\left[\begin{array}{ccccccccc}
0.0201 & -60.2970 & -2.3970 & 41.4615 & 0.0379 & 0.0070 & -6.7544 & -0.0144 & 7.9662 \\
0.0958 & -63.3253 & -1.2661 & 43.8057 & -0.2507 & -0.0376 & -7.0918 & 0.0008 & 8.1504 \\
-0.0903 & -63.3167 & -1.2659 & 43.8002 & 0.2715 & 0.0418 & -7.0908 & -0.0163 & 8.1494 \\
-0.2389 & -25.1090 & 0.3741 & 17.5555 & -0.4580 & -0.0825 & -2.8105 & 0.1068 & 3.0808 \\
0.2608 & -25.1480 & 0.3732 & 17.5802 & 0.4975 & 0.0899 & -2.8151 & -0.1209 & 3.0853 \\
0.0987 & -3.8486 & -0.0060 & 0.2298 & 0.4124 & 0.0432 & -0.4189 & 0.0660 & 0.5860 \\
-0.0982 & -3.8464 & -0.0060 & 0.2284 & -0.4107 & -0.0432 & -0.4184 & -0.0667 & 0.5856
\end{array}\right] .
$$
Figure 3.

In order to compare results, the adaptive FTC in our research and a normal $H$-infinity tracking controller as [15], which do not consider fault and saturation, are both carried out in simulations. Here, the unknown external disturbance is assumed as $w(t)=[0.01 \cos t, 0,0,-0.02 \sin t$, $0,0.015 \cos t, 0.01 \sin t, 0,0]^{T}$. When no actuator faults and saturation occur, the adaptive controller in Theorem 5 becomes a normal $H$-infinity tracking controller and can guarantee that the outputs of the flight control systems of UAV accurately track command within three seconds as

To verify the excellent fault tolerant control capability of presented method in this study, let the following two actuator channels exhibit faults; that is,

$$
\begin{aligned}
& \rho_{1}(t)= \begin{cases}1, & t<t_{f} \\
0.8, & t \geq t_{f}\end{cases} \\
& \rho_{5}(t)= \begin{cases}1, & t<t_{f} \\
0.4, & t \geq t_{f},\end{cases}
\end{aligned}
$$

where $t_{f}=5 \mathrm{~s}$ is the occurrence time of faults.

When the flight control systems (FCS) of UAV only occur unknown actuator faults; namely, $\rho_{1}=80 \%, \rho_{5}=40 \%$, where $\rho_{1}$ is the control effectiveness of actuator channel $\delta_{\text {th }}$ and $\rho_{5}$ is the control effectiveness of actuator channel $\delta_{\mathrm{fl}}$. In Figure 4 , the dashed line outputs of UAV produced by the normal $H$-infinity tracking controller without FTC do not track the given command. On the other hand, using the adaptive fault accommodation approach developed in our research, all the outputs of FCS denoted by solid line can asymptotically converge to $y_{r}$. Simultaneously, the estimation values $\widehat{\rho}_{s}, s=1,5$ asymptotically converge to actual control effectiveness of $\delta_{\text {th }}$ and $\delta_{\mathrm{fl}}$; namely, $\hat{\rho}_{1} \rightarrow 0.8, \widehat{\rho}_{5} \rightarrow 0.4$ as displayed in Figure 5.

To verify the adaptive capability of proposed approach in saturation case, the control surface position limits, namely, actuator saturation levels are defined as $\delta_{1 \min }=0(\mathrm{deg}), \delta_{i \min }=$ $-5(\mathrm{deg})(i=2, \ldots, 7)$, and $\delta_{i \max }=5(\mathrm{deg})(i=1, \ldots, 7)$. At 

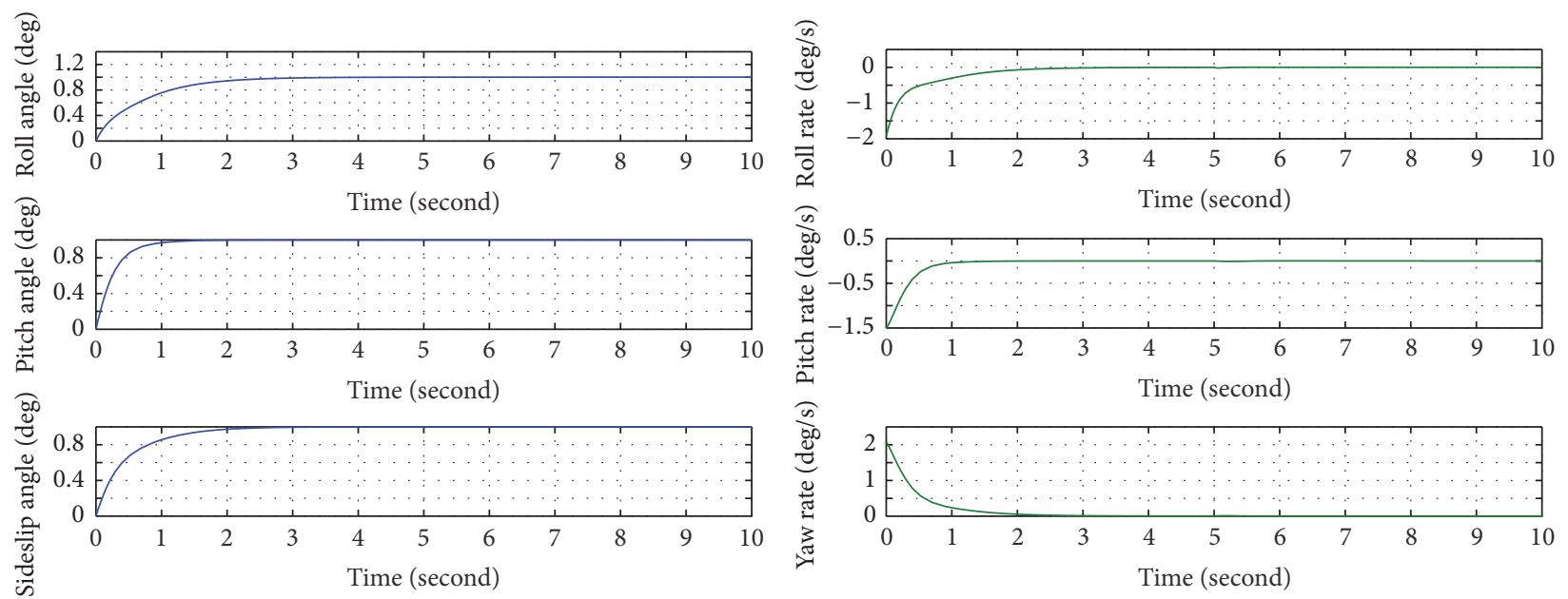

FIGURE 3: The output curves of FCS in healthy case.
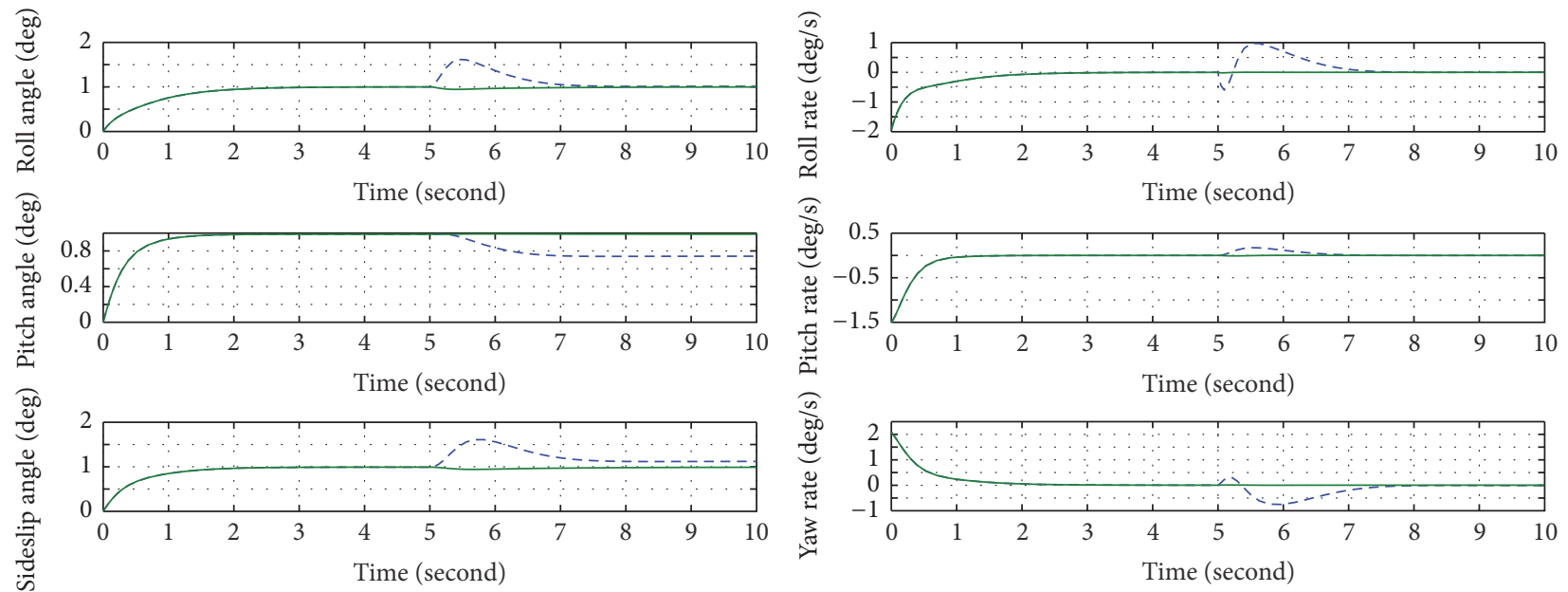

FIGURE 4: The output curves of FCS in faulty case.
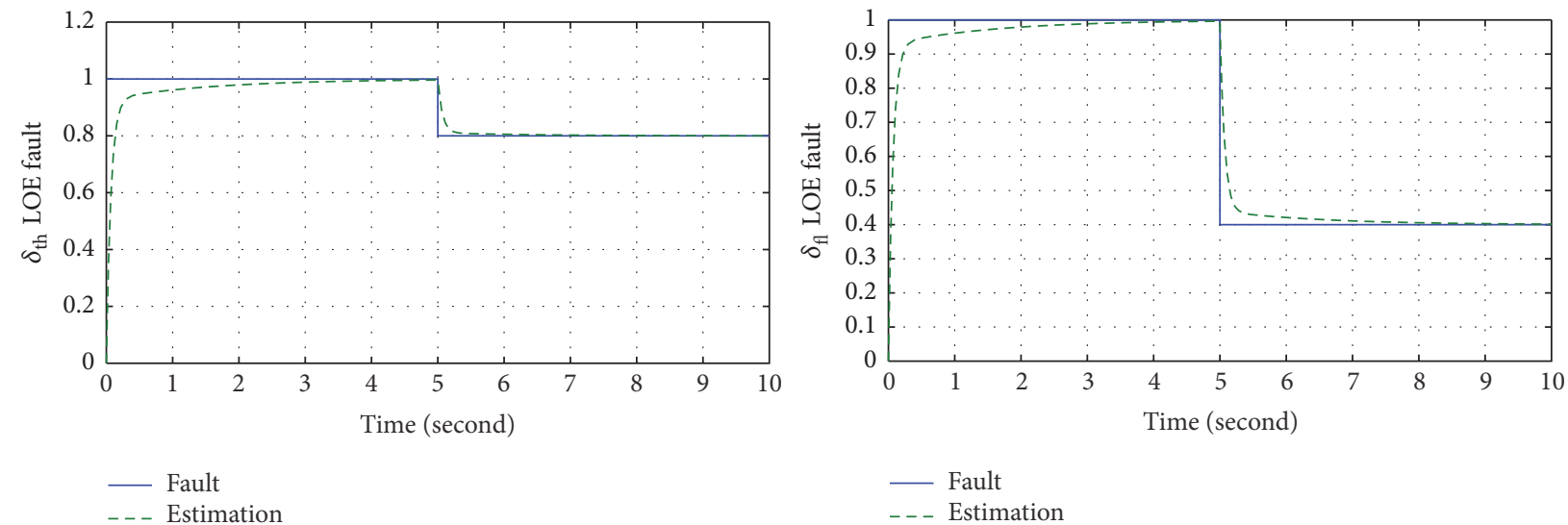

FIGURE 5: The fault estimation for throttle and left flap in faulty case. 

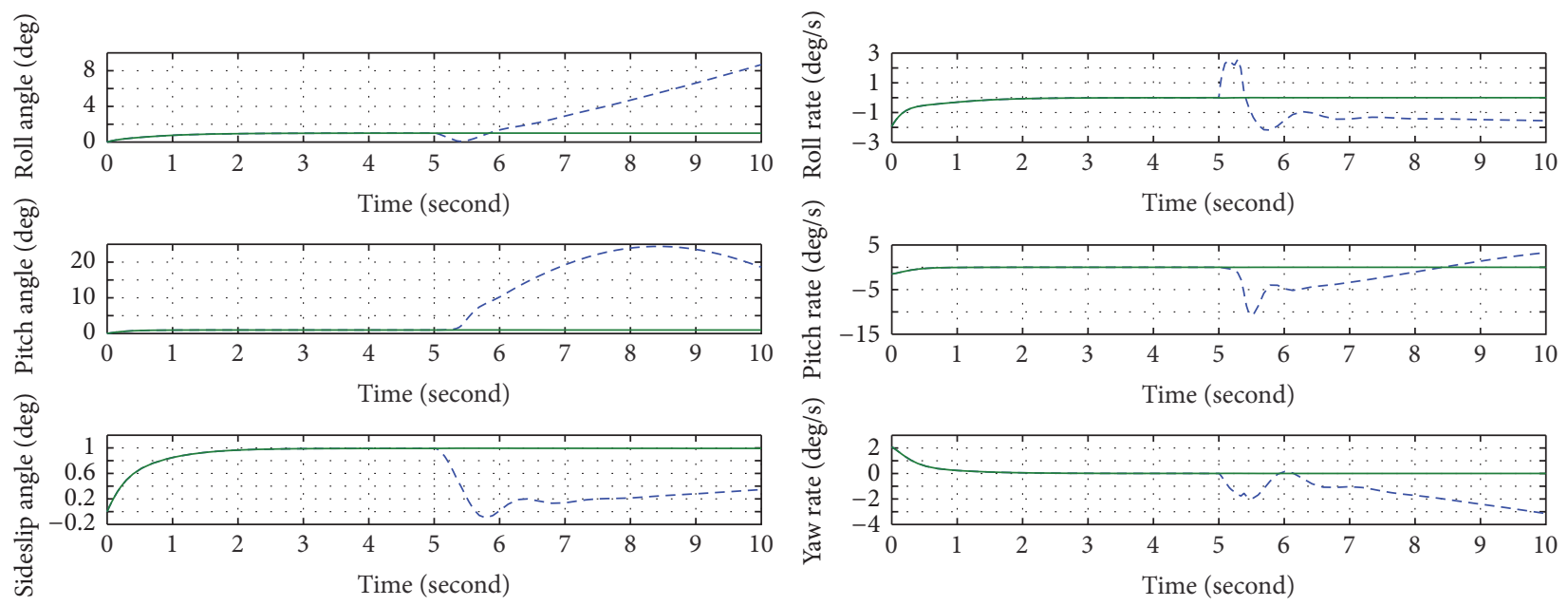

FIgURE 6: The output curves of FCS in faulty and saturated case.

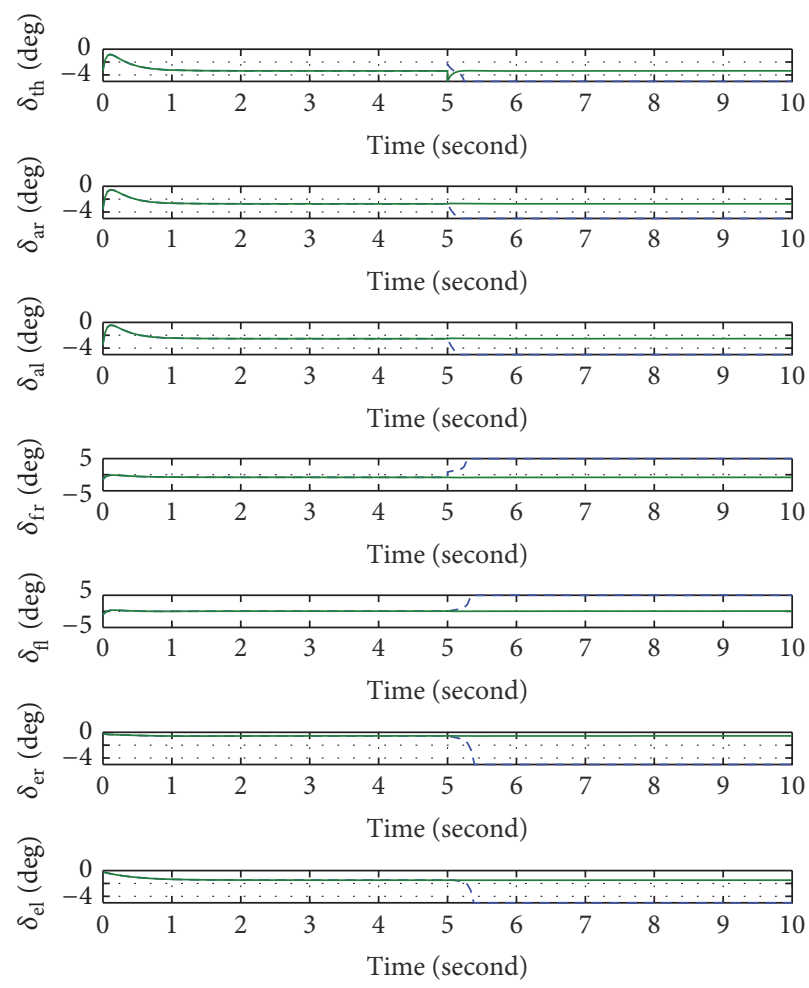

FIGURE 7: The input curves of FCS in faulty and saturated case.

$t=5$ (s), the above actuator faults similarly occur. According to Figure 6, we observe that satisfactory tracking performance of FCS with FTC, which compensates the actuator faults and saturation, is obtained. At the same time, it is easily seen that the outputs of FCS using the normal $\mathrm{H}$-infinity tracking controller without FTC are unstable. Moreover, the control input responses of this controller with FTC are smaller than the ones of the normal controller without FTC; therefore less energy is required by this FTTC as shown in Figure 7.

\section{Conclusion}

This research addresses a fault tolerant tracking controller (FTTC) approach for flight control systems of an UAV with actuator saturation and faults. The T-S fuzzy models are employed for representing FCS of an UAV. Considering saturation constraints, unknown LOE faults, and disturbance, a novel FTTC strategy is developed by adaptive theory. On the basis of Lyapunov technique, the stability of the T-S fuzzy 
FCS is proved. Finally, the simulation illustrates the efficiency of the presented FTTC. In fact, the T-S fuzzy model used in our study is an approximative model of UAV; we would study the general nonlinear flight control systems model of UAV in future research.

\section{Competing Interests}

The authors declare that they have no competing interests.

\section{Acknowledgments}

This work was partially supported by the National Natural Science Foundation of China (61403195), the Natural Science Foundation of Jiangsu Province, China (SBK2014042586), and the Fundamental Research Funds for the Central Universities (NJ20160051).

\section{References}

[1] Y. J. Xu, "Nonlinear robust stochastic control for unmanned aerial vehicles," in Proceedings of the 2009 American Control Conference (ACC '09), pp. 2819-2824, St. Louis, Mo, USA, June 2009.

[2] Z. Liu and Y. Wang, "Fuzzy adaptive tracking control within the full envelope for an unmanned aerial vehicle," Chinese Journal of Aeronautics, vol. 27, no. 5, pp. 1273-1287, 2014.

[3] Y. Zhang and J. Jiang, "Bibliographical review on reconfigurable fault-tolerant control systems," Annual Reviews in Control, vol. 32, no. 2, pp. 229-252, 2008.

[4] F. Bateman, H. Noura, and M. Ouladsine, "Fault diagnosis and fault-tolerant control strategy for the aerosonde UAV," IEEE Transactions on Aerospace and Electronic Systems, vol. 47, no. 3, pp. 2119-2137, 2011.

[5] T. Takagi and M. Sugeno, "Fuzzy identification of systems and its applications to modeling and control," IEEE Transactions on Systems, Man and Cybernetics, vol. 15, no. 1, pp. 116-132, 1985.

[6] X. Sun, Y. Gao, and C. Wu, "Output tracking control for a class of continuous-time T-S fuzzy systems," Neurocomputing, vol. 152, pp. 199-208, 2015.

[7] S. Tong, T. Wang, and W. Zhang, "Fault tolerant control for uncertain fuzzy systems with actuator failures," International Journal of Innovative Computing, Information and Control, vol. 4, no. 10, pp. 2461-2474, 2008.

[8] H. Ghorbel, A. El Hajjaji, M. Souissi, and M. Chaabane, "Faulttolerant trajectory tracking control for Takagi-Sugeno systems with unmeasurable premise variables: descriptor approach," Circuits, Systems, and Signal Processing, vol. 33, no. 6, pp. 17631781, 2014.

[9] M. S. Shaker and R. J. Patton, "Active sensor fault tolerant output feedback tracking control for wind turbine systems via T-S model," Engineering Applications of Artificial Intelligence, vol. 34, pp. 1-12, 2014.

[10] H. Yang, P. Shi, X. Li, and Z. Li, "Fault-tolerant control for a class of T-S fuzzy systems via delta operator approach," Signal Processing, vol. 98, pp. 166-173, 2014.

[11] S. Wang, Y. Jiang, Y. Li, and D. Liu, "Reliable observer-based $H_{\infty}$ control for discrete-time fuzzy systems with time-varying delays and stochastic actuator faults via scaled small gain theorem," Neurocomputing, vol. 147, no. 1, pp. 251-259, 2015.

[12] B. Chen, X.-P. Liu, S.-C. Tong, and C. Lin, "Observer-based stabilization of T-S fuzzy systems with input delay," IEEE Transactions on Fuzzy Systems, vol. 16, no. 3, pp. 652-663, 2008.
[13] Y.-H. Wang, Q.-X. Wu, C.-S. Jiang, and G.-Y. Huang, "Reentry attitude tracking control based on fuzzy feedforward for reusable launch vehicle," International Journal of Control, Automation and Systems, vol. 7, no. 4, pp. 503-511, 2009.

[14] H. H. Wang and M. Wang, "Robust fuzzy variable structure control of T-S model for a quadrotor unmanned air vehicle," International Journal of Multimedia and Ubiquitous Engineering, vol. 10, no. 5, pp. 277-286, 2015.

[15] B. Mansouri, N. Manamanni, K. Guelton, A. Kruszewski, and T. M. Guerra, "Output feedback LMI tracking control conditions with $H_{\infty}$ criterion for uncertain and disturbed T-S models," Information Sciences, vol. 179, no. 4, pp. 446-457, 2009.

[16] H. J. Gao, Y. Zhao, and T. W. Chen, " $H_{\infty}$ fuzzy control of nonlinear systems under unreliable communication links," IEEE Transactions on Fuzzy Systems, vol. 17, no. 2, pp. 265-278, 2009.

[17] P. Shi, Y. Zhang, M. Chadli, and R. K. Agarwal, "Mixed Hinfinity and passive filtering for discrete fuzzy neural networks with stochastic jumps and time delays," IEEE Transactions on Neural Networks and Learning Systems, vol. 27, no. 4, pp. 903909, 2016.

[18] X. Su, P. Shi, L. Wu, and M. V. Basin, "Reliable filtering with strict dissipativity for T-S fuzzy time-delay systems," IEEE Transactions on Cybernetics, vol. 44, no. 12, pp. 2470-2483, 2014.

[19] F. Li, P. Shi, L. Wu, and X. Zhang, "Fuzzy-model-based Dstability and nonfragile control for discrete-time descriptor systems with multiple delays," IEEE Transactions on Fuzzy Systems, vol. 22, no. 4, pp. 1019-1025, 2014.

[20] H. Li, H. Liu, H. Gao, and P. Shi, "Reliable fuzzy control for active suspension systems with actuator delay and fault," IEEE Transactions on Fuzzy Systems, vol. 20, no. 2, pp. 342-357, 2012.

[21] S. Tong, Y. Xu, and Y. Li, "Adaptive fuzzy decentralised control for stochastic nonlinear large-scale systems in pure-feedback form," International Journal of Systems Science, vol. 46, no. 8, pp. 1510-1524, 2015.

[22] S. Tong, S. Sui, and Y. Li, "Adaptive fuzzy decentralized output stabilization for stochastic nonlinear large-scale systems with unknown control directions," IEEE Transactions on Fuzzy Systems, vol. 22, no. 5, pp. 1365-1372, 2014.

[23] D. Zhang, Q.-L. Han, and X. Jia, "Network-based output tracking control for T-S fuzzy systems using an event-triggered communication scheme," Fuzzy Sets and Systems, vol. 273, pp. 26-48, 2015.

[24] B. Kadmiry and D. Driankov, "A fuzzy gain-scheduler for the attitude control of an unmanned helicopter," IEEE Transactions on Fuzzy Systems, vol. 12, no. 4, pp. 502-515, 2004.

[25] Y. Chen, T. M. Wang, J. H. Liang, C. L. Wang, and C. Xue, "A fuzzy robust path following controller for a small unmanned air vehicle," in Proceedings of the 7th IEEE Conference on Industrial Electronics and Applications (ICIEA '12), pp. 11891194, Singapore, July 2012.

[26] S. A. Salman, A. G. Sreenatha, and J. Y. Choi, "Indirect adaptive fuzzy control of unmanned aerial vehicle," in Proceedings of the 17th World Congress, International Federation of Automatic Control (IFAC '08), pp. 13229-13234, Seoul, Republic of Korea, July 2008.

[27] Y. C. Ma, M. H. Chen, and Q. L. Zhang, "Memory dissipative control for singular T-S fuzzy time-varying delay systems under actuator saturation," Journal of the Franklin Institute, vol. 352, no. 10, pp. 3947-3970, 2015.

[28] D. Ye and G.-H. Yang, "Adaptive fault-tolerant tracking control against actuator faults with application to flight control," IEEE Transactions on Control Systems Technology, vol. 14, no. 6, pp. 1088-1096, 2006. 


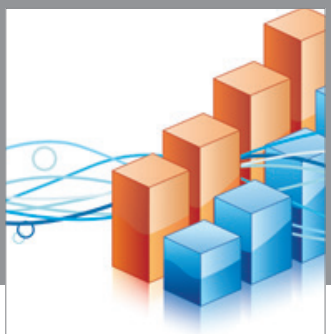

Advances in

Operations Research

vatem alat4

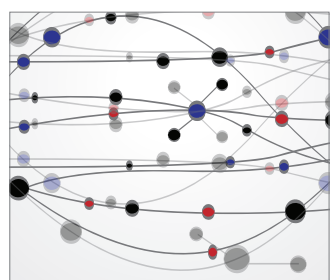

\section{The Scientific} World Journal
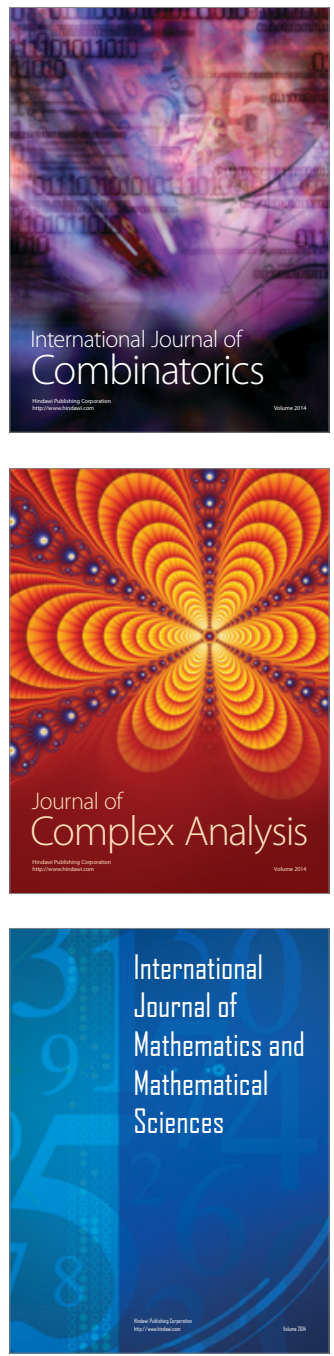
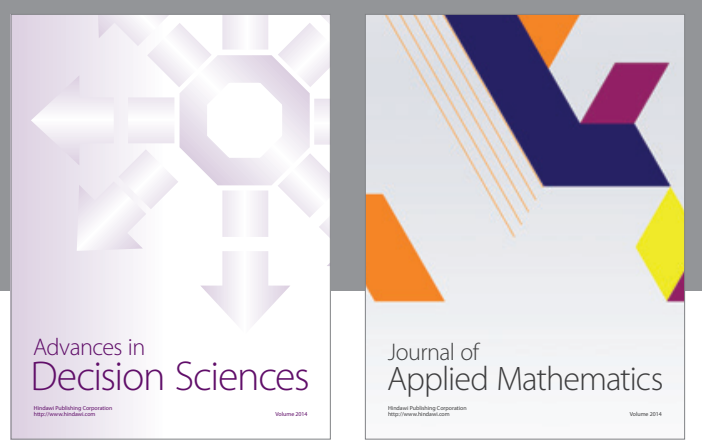

Algebra

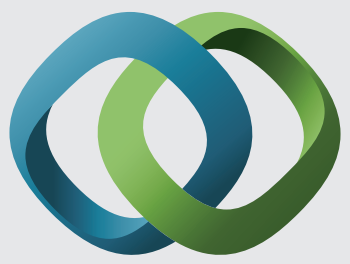

\section{Hindawi}

Submit your manuscripts at

http://www.hindawi.com
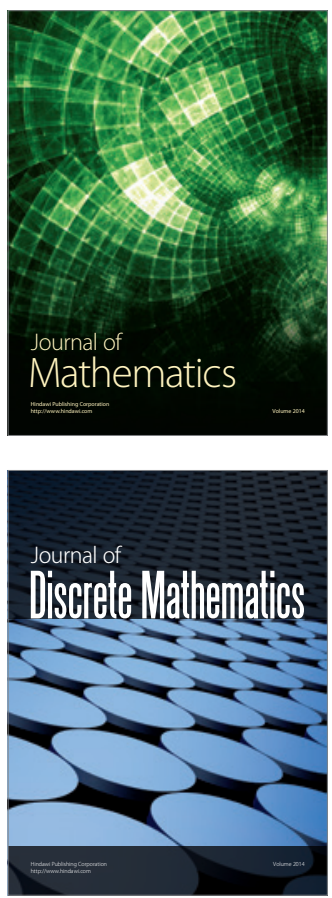

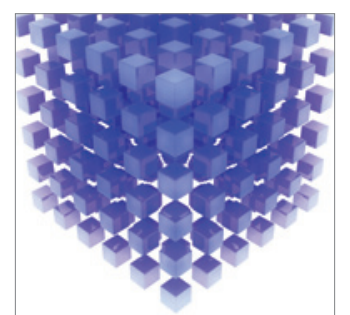

Mathematical Problems in Engineering
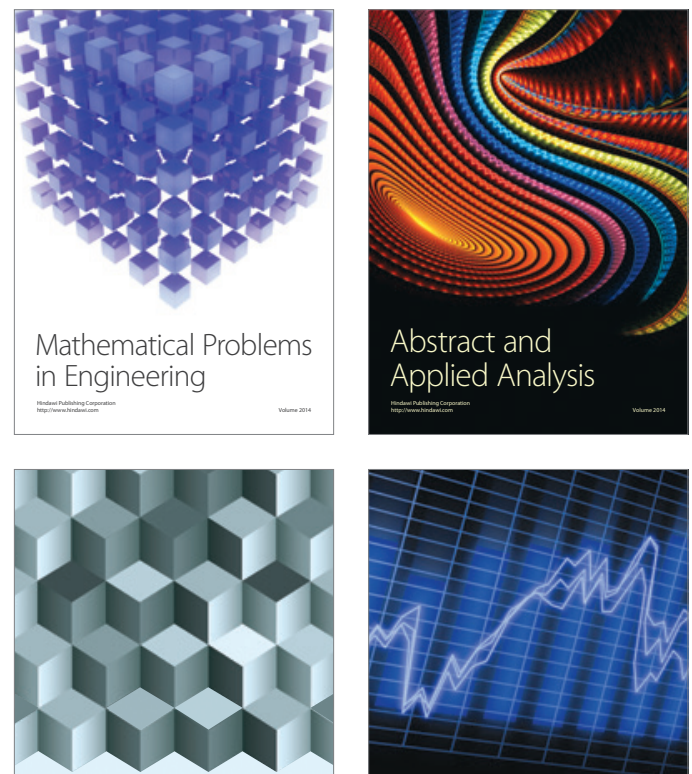

Journal of

Function Spaces

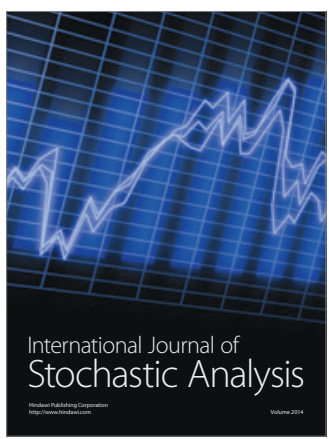

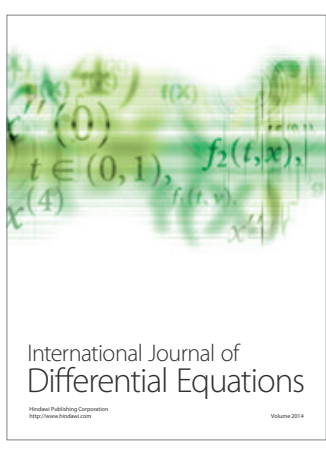
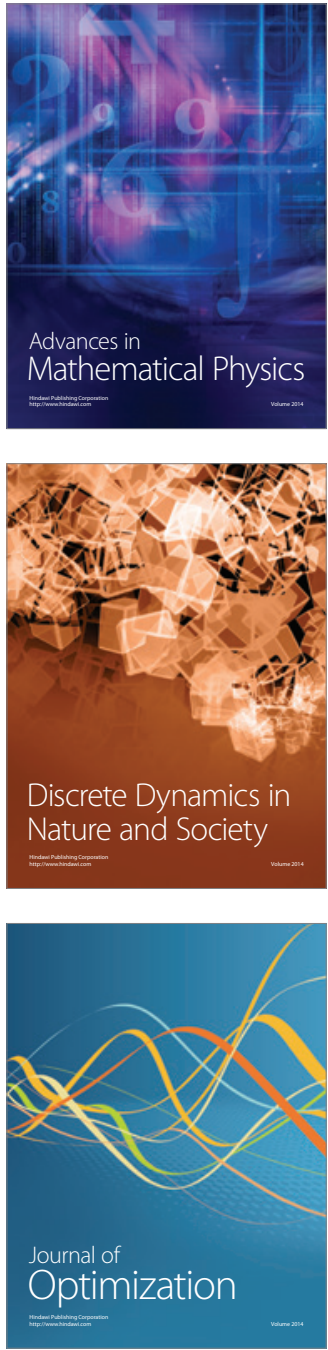